\title{
In silico Evaluation of PLAC1-fliC As a Chimeric Vaccine against Breast Cancer
}

\author{
Mortaza Taheri-Anganeh ${ }^{1}$, Ahmad Amiri ${ }^{2}$, Ahmad Movahedpour ${ }^{1}$, Seyyed Hossein Khatami ${ }^{2}$, \\ Younes Ghasemi ${ }^{3,4}$, Amir Savardashtaki ${ }^{1,4}$ and Zohreh Mostafavi-Pour ${ }^{2,5^{*}}$
}

\footnotetext{
${ }^{1}$ Department of Medical Biotechnology, School of Advanced Medical Sciences and Technologies, Shiraz University of Medical Sciences, Shiraz, Iran; ${ }^{2}$ Department of Biochemistry, School of Medicine, Shiraz University of Medical Sciences, Shiraz, Iran; ${ }^{3}$ Department of Pharmaceutical Biotechnology, School of Pharmacy, Shiraz University of Medical Sciences, Shiraz, Iran; ${ }^{4}$ Pharmaceutical Sciences Research Center, Shiraz University of Medical Sciences, Shiraz, Iran

${ }^{5}$ Maternal-Fetal Medicine Research Center, Shiraz University of Medical Sciences, Shiraz, Iran
}

Received 10 June 2019; accepted 24 June 2019; published online 18 November 2019

\begin{abstract}
Background: Breast cancer is one of the most prevalent cancers among women. Common cancer treatment methods are not effective enough, and there is a need for a more efficient treatment procedure. Cancer vaccine is a novel immunotherapy method that stimulates humoral and/or cellular immunity against cancer. PLAC1 is a cancer/testis antigen, prevalent in breast cancer and rarely found in normal tissues. FliC, as a bacterial adjuvant, when fused to PLAC1 can elicit humoral and cellular responses. Therefore, PLAC1-fliC is a chimeric protein, which can be considered a suitable candidate against breast cancer. Methods: ProtParam was used to evaluate the physicochemical properties of PLAC1-fliC. Second structures were determined using the GOR V server. PLAC1-fliC 3D structure was modeled by Phyre2, and it was refined using GalaxyWEB. The refined model was submitted to RAMPAGE, PROCHECK, and PrOSA-web for validation. Antigenicity and allergenicity of the construct were predicted by ANTIGENpro, VaxiJen, AllergenFP, and SDAP databases. Then MHC-I- and MHC-II-binding epitopes of PLAC1-fliC were forecasted by NetMHC 4.0 and NetMHCII 2.3 Servers. Finally, Ellipro and CTLpred were employed to predict B-cell and CTL epitopes. Results: The construct was evaluated as a stable fusion protein, which could be antigenic and could stimulate B and T cells against breast cancer. Conclusion: PLAC1-flic, as a cancer vaccine candidate, might be suitable and specific for breast cancer, which could evoke humoral and cellular immunity against this type of tumor. DOI: 10.29252/ibj.24.3.173
\end{abstract}

Keywords: Bioinformatics, Breast cancer, Cancer vaccines, PLAC1

Corresponding Author: Zohreh Mostafavi-Pour

Department of Biochemistry, School of Medicine, Shiraz University of Medical Sciences, Shiraz, Iran; Tel. \& Fax.: (+98-713) 2303029;

E-mail: zmostafavipour88@yahoo.co.uk

\section{INTRODUCTION}

$\mathrm{B}$ reast cancer is the most frequently diagnosed cancer and cancer-related death amongst women. Annually, more than two million breast cancer cases are reported worldwide ${ }^{[1]}$. Despite the conventional therapeutic modalities for breast cancer treatment, these therapies have been suggested to be rather ineffective ${ }^{[2]}$.

New studies have shown that immunotherapy can be an effective and alternative choice in treating breast cancer ${ }^{[3]}$. Immunotherapy consists of anti-cancer antibody, cancer vaccine, and T-cell receptor engineering ${ }^{[2]}$. PLAC1 is a cancer/testis antigen with a significant role in cancer progression and invasion ${ }^{[4]}$. Cancer/testis antigens are specific antigens expressed 
in germ-line cells like testis, fetal ovary, and placenta. These antigens are also expressed in some cancer cells, but rarely found in normal tissues ${ }^{[5]}$. Studies have revealed that PLAC1 expression in normal cells is not adequate to be measured, while its expression in cancer cells is measurable ${ }^{[5,6]}$. Recently, it has been displayed that PLAC1 plays a critical role in tumor invasion and metastasis through Furin/NICD/PTEN/AKT axis ${ }^{[7]}$. PLAC1 is a type II membrane bound protein, which its 5-22 amino acids form a transmembrane helix, and has a large extracellular domain consisting of 23-212 amino acids. A truncated zona pellucida domain in the extracellular part of PLAC1 is made of amino acids 29$119^{[8]}$. New research on PLAC1 has indicated that it is well expressed in a versatile cancer cases, specifically in breast cancer, but not expressed in normal tissues, except testis ${ }^{[9]}$. Therefore, PLAC1 could be a specific target for breast cancer immunotherapy.

Vaccines need an adjuvant to be more effective ${ }^{[10]}$. Adjuvants induce TLR, which results in helper T-cell activation. Bacterial flagellin is one of the most important protein adjuvants that induces TLR5 receptors ${ }^{[11]}$. FliC protein is the main component of flagellin and encoded by flic gene ${ }^{[12]}$. Salmonella enterica serovar typhimurium FliC, as an efficient adjuvant, is widely used in vaccine research ${ }^{[13]}$. FliC is made of four domains, including D0, D1, D2, and D3. D1 is responsible for TLR5 binding and dimerization of TLR5s and triggers the downstream signaling and stimulates cells to secrete proinflammatory cytokines such as TNF- $\alpha^{[14]}$. Flagellin is a TLR5 binding ligand and starts downstream signaling through MyD88 pathway, which activates innate immunity. It has been shown that the innate immune system motivation results in cytokines secretion and dendritic cells $\operatorname{activation}^{[15]}$.

The aim of this study was to design a fusion protein construct, as an effective vaccine, consisting of PLAC1 (as a specific antigen) and Salmonella enterica fliC (as a bacterial adjuvant) that can stimulate humoral and cellular immune responses against breast cancer. This construct was evaluated using bioinformatics online web servers.

\section{MATERIALS AND METHODS}

\section{Construct design}

In this study, the amino acid sequences of PLAC1 and fliC were extracted from Uniprot database (https://www.uniprot.org/) in FASTA format (Uniprot id: Q9HBJ0). Amino acids 23-212 of PLAC1 were considered for the construct design, and residues 1-22 were neglected because they are located in plasma membrane and cytosol, and the humoral immunity does not have access to them. A flexible linker (GSGGSGGSGGSG) was located between PLAC1 antigen and fliC adjuvant. Our final construct was PLAC1 (23-212)-linker (GSGGSGGSGGSG)-fliC.

\section{Prediction of physicochemical properties and secondary structure}

To predict different physicochemical features, such as instability index, isoelectric point, aliphatic index, grand average of hydropathicity, and molecular weight for PLAC1-fliC, we utilized ProtParam server (https://web.expasy.org/protparam/) ${ }^{[16]}$. Secondary structure of PLAC1, fliC, and PLAC1-fliC were predicted using GOR V server (https://abs.cit.nih.gov/ gor/) and were compared together ${ }^{[17]}$.

\section{Tertiary structure prediction and refinement}

Phyre2 server (http://www.sbg.bio.ic.ac.uk/ phyre2/ html/page.cgi?id=index) was employed to predict the $3 \mathrm{D}$ structure of the construct based on homology modeling method ${ }^{[18]}$. The model was refined using GalaxyRefine server (http://galaxy. seoklab.org/cgibin/submit.cgi?type=REFINE) ${ }^{[19]}$, and the best refined model was selected and submitted for next steps.

\section{Validation of tertiary structure}

For the validation of the model, the following servers were used: RAMPAGE (http://servicesn.mbi.ucla.edu/ PROCHECK/), ProSA-web (https://prosa.services. came.sbg.ac.at/prosa.php), and PROCHECK (http:// servicesn.mbi.ucla.edu/PROCHECK/). RAMPAGE server has ability to check stereochemical qualities of the models peptide bonds and shows the number of residues in favored, allowed and outer areas in a Ramachandran plot. ProSA-web has a diagnostic method that is able to analyze protein structures based on all the available protein structures ${ }^{[20]}$. PROCHECK server was employed for evaluating the stereochemical quality of the PLAC1-fliC ${ }^{[21]}$. The results of all the three servers were compared before and after $3 \mathrm{D}$ structure model refinement.

\section{Antigenicity and allergenicity prediction}

VaxiJen server (http://www.ddg-pharmfac.net/ vaxijen/VaxiJen/ VaxiJen.html) was used for the prediction of protective antigens and subunit vaccines. According to the physicochemical properties of proteins, this server classifies antigens without referral to sequence alignment. The accuracy of the server based on the origin of the protein (bacterial, viral, and tumor protein datasets) varies between 70 and $89 \%{ }^{[22]}$. Antigenicity of the construct was rechecked by ANTIGENpro (http://scratch.proteomics.ics.uci.edu/), 


\section{QSPMTVLCSIDWFMVTVHPFMLNNDVCVHFHELHLGLGCPPNHVQPHAY QFTYRVTECGIRAKAVSQDMVIYSTEIHYSSKGTPSKFVIPVSCAAPQKSPW LTKPCSMRVASKSRATAQKDEKCYEVFSLSQSSQRPNCDCPPCVFSEEEHQ VPCHQAGAQEAQPLQPSHFLDISEDWSLHTDDMIGSMGSGGSGGSGGSGM AQVINTNSLSLLTQNNLNKSQSALGTAIERLSSGLRINSAKDDAAGQAIAN RFTANIKGLTQASRNANDGISIAQTTEGALNEINNNLQRVRELAVQSANST NSQSDLDSIQAEITQRLNEIDRVSGQTQFNGVKVLAQDNTLTIQVGANDGE TIDIDLKQINSQTLGLDTLNVQQKYKVSDTAATVTGYADTTIALDNSTFKA SATGLGGTDQKIDGDLKFDDTTGKYYAKVTVTGGTGKDGYYEVSVDKTN GEVTLAGGATSPLTGGLPATATEDVKNVQVANADLTEAKAALTAAGVTG TASVVKMSYTDNNGKTIDGGLAVKVGDDYYSATQNKDGSISINTTKYTAD DGTSKTALNKLGGADGKTEVVSIGGKTYAASKAEGHNFKAQPDLAEAAT TTENPLQKIDAALAQVDTLRSDLGAVQNRFNSAITNLGNTVNNLTSARSRI EDSDYATEVSNMSRAQILQQAGTSVLAQANQVPQNVLSLLR}

Fig. 1. Amino acid composition of PLAC1-fliC construct. Red shows PLAC1, black indicates a flexible linker, and green shows fliC.

which is based on pathogen independent, sequencebased, alignment-free analysis and uses antigenicity microarray data for predicting the protein antigenicity. SDAP (http://fermi.utmb.edu/SDAP/sdap_man.html) and AllergenFP (http://www.ddgpharmfac.net/ AllergenFP/) databases were employed for allergenicity prediction of the fusion protein. SDAP is the structural database of allergenic proteins, while AllergenFP online bioinformatics tool is based on descriptor fingerprint ${ }^{[23]}$.

\section{MHC-I and MHC-II binding epitope prediction}

NetMHC 4.0 Server (http://www.cbs.dtu.dk/services/ NetMHC/) and NetMHCII 2.3 Server (http://www.cbs. dtu.dk/services/NetMHCII/) were applied to predict MHC-I binding epitopes (based on an artificial neural networks method) and MHC-II binding epitopes, respectively ${ }^{[24,25]}$.

\section{$B$ cell and CTL epitopes prediction}

B-cell epitopes, both continuous and discontinuous, were predicted using ElliPro server (http://tools. iedb.org/ellipro/) ${ }^{[26]}$. CTLPred server (http://crdd.osdd. net/raghava/ctlpred/) was utilized for the prediction of CTL epitopes based on the direct method. This method uses data on T-cells epitopes templates instead of MHC-binding peptides. CTLpred method is based on techniques such as artificial neural network and support vector $^{[27]}$.

\section{RESULTS}

\section{Physicochemical properties}

The fusion construct was designed (Fig. 1). The predicted molecular weight and isoelectric point for our fusion protein were 73642.54 Da and 5.17, respectively. Total numbers of negatively and positively charged amino acids of this protein were 73 and 55; thus, its final charge was -18 , and it totally had a negative charge. Instability index for PLAC1-fliC protein was 32.87 , which was considered as the stable protein; the instability index under 40 means stability. Aliphatic index and grand average of hydropathicity of our fusion protein were 76.85 and -0.380 , respectively.

\section{Secondary structure}

Our findings showed that PLAC1-fliC had $29.31 \%$ alpha helix, $21.98 \%$ extended strand, and $48.71 \%$ random coil. Comparison between PLAC1, fliC, and PLAC1-fliC is shown in Table 1 , and the secondary structure pattern of PLAC1-fliC is indicated in Figure 2.

\section{Homology model building, refinement, and validation}

The PLAC1-fliC protein model was built using Phyre2 based on Hidden Markov Model. Figure 3 depicts fusion protein predicted tertiary structure. This model was refined using GalaxyRefine server, and five

Table 1. Comparison of PLAC1, fliC, and PLAC1-fliC fusion secondary structures

\begin{tabular}{lccc}
\hline Protein & Alpha helix (\%) & Extended strand (\%) & Random coil (\%) \\
\hline PLAC1 & 5.26 & 36.32 & 58.42 \\
fliC & 39.27 & 16.19 & 44.53 \\
PLAC1-fliC & 29.31 & 21.98 & 48.71 \\
\hline
\end{tabular}



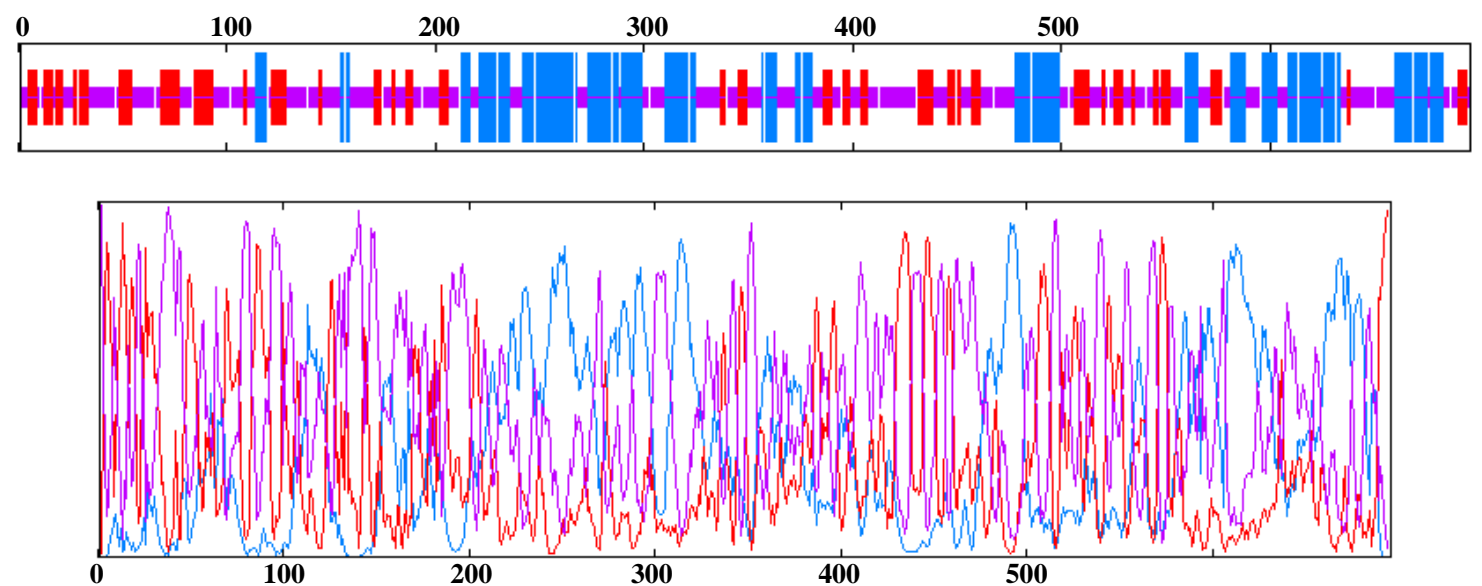

Fig. 2. Graphical results of secondary structures comparison. Helix, extended strand, and random coil are indicated by blue, purple and red, respectively.

refined models were built, and the best one was chosen. ProSA Z-scores for PLAC1-fliC 3D model after and before refinement were -8.38 and -9.21 , respectively (Fig. 4). RAMPAGE results showed that the numbers of residues increased in the favored region after the model refinement. These findings were confirmed by PROCHECK results (Table 2).

\section{Antigenicity and allergenicity}

ANTIGENpro and VaxiJen results revealed that fliC, as an adjuvant, could increase the antigenicity of PLAC1 protein. Table 3 shows the antigenicity of PLAC1, fliC, linker, and PLAC1-fliC fusion protein. In accordance with AllergenFP server, our findings suggested that PLAC1, fliC, and PLAC1-fliC could not be an allergen for human body. Searching PLAC1, fliC, and PLAC1-fliC sequences in SDAP database showed that these sequences were not allergens, and, therefore, AllergenFP results were confirmed.

\section{MHC-I and MHC-II-binding epitopes}

Since 9-mer peptides display more tendency to HLA1, peptides with nine amino acids were chosen. The 9-mer peptides with strong binding preference to HLA-A0201 subtype with their affinity and rank are shown in Table 4. To predict MHC-II binding, 15-mer peptides binding to DRB1_ 0301, DRB1_ 0401, DRB1_ 0701, and DRB1_ 1501 were evaluated. Our finding exhibited that PLAC1-fliC epitopes were strong binders to DRB1_ 0301 and DRB1_ 0401 (Table 5).

\section{$B$ cell and CTL epitopes}

Using ElliPro, B cell specific continuous (linear) and discontinuous (conformational) epitopes were defined. Accordingly, 16 continuous epitopes were identified, and their length was between 6-110 amino acids (Table 6). In addition, 4 discontinuous epitopes were predicted with maximum 177 and minimum 15 amino acids in length (Table 7). Top 10 CTL peptide epitopes with higher scores predicted by combined approach, artificial neural network/support vector machine, are shown in Table 8.

\section{DISCUSSION}

According to the global cancer statistics in 2018, breast cancer is the most commonly diagnosed and the leading cause of cancer-related death among women ${ }^{[1]}$. However, there are merely few therapeutic strategies with limited success against breast cancer ${ }^{[28]}$. One new therapeutic strategy against cancer is immunotherapy. Cancer vaccine is one of the most effective immunotherapy treatments, but its design and production is often time-consuming and costly. The new generation of vaccines are based on recombinant

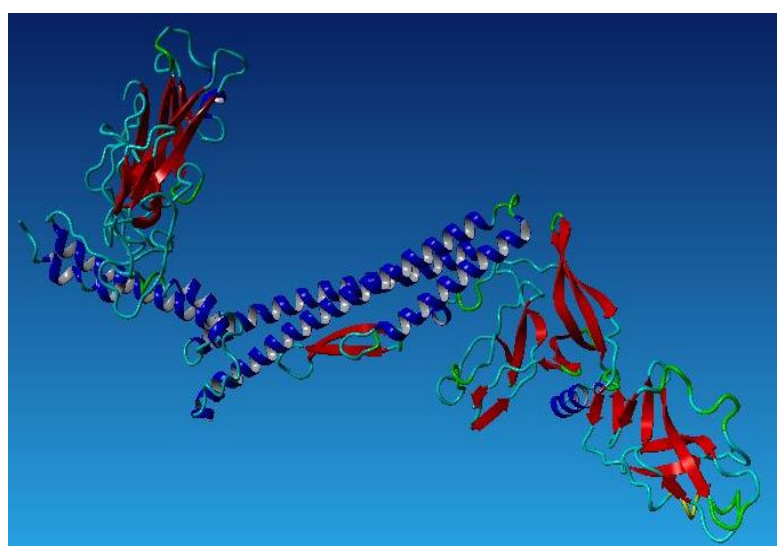

Fig. 3. The 3D structure of PLAC1-fliC defined by Phyre 2 . 

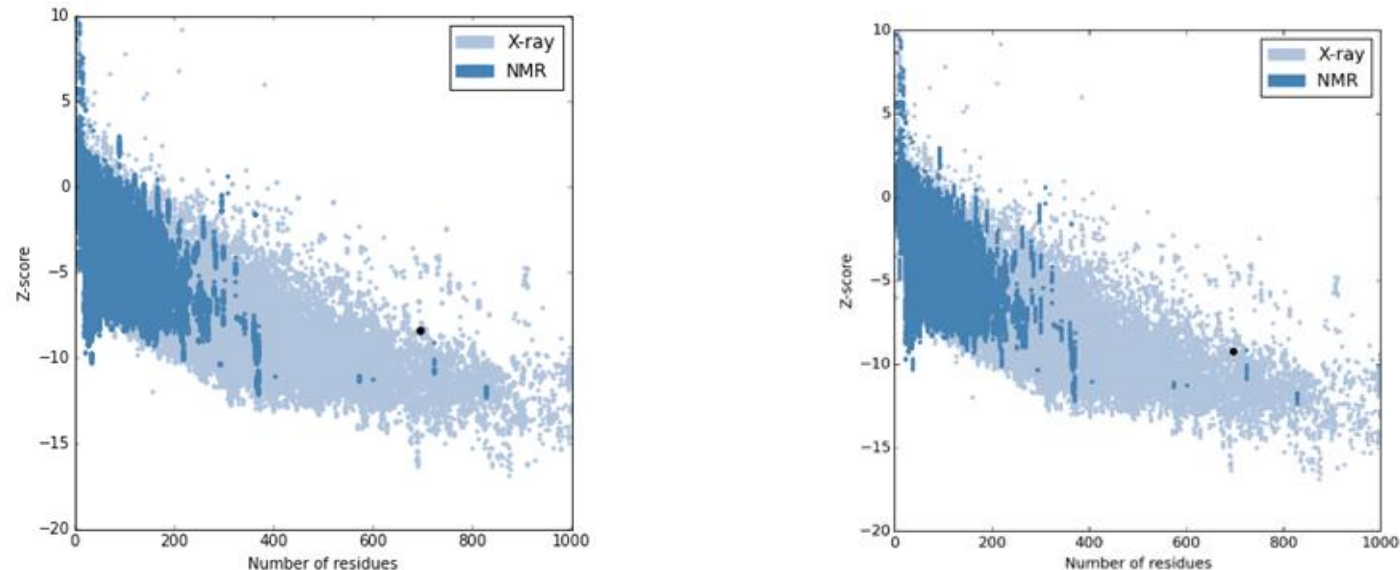

Fig. 4. The Z-score plot for the 3D model of PLAC1-fliC before (A) and after (B) refinement. Z-score is shown by a black spot.

protein technology according to their specific design, safety, purity, and feasibility of production ${ }^{[29]}$. Thus, finding and screening the putative vaccine candidate is a prerequisite for its production. In a previous study, Kawada et al. ${ }^{[30]}$ vaccinated 13 patients using NYESO-1, a cancer-testis antigen. This antigen induces antibody production, as well as CD4 and CD8 T-cell responses. Furthermore, Shim et al. ${ }^{[31]}$ showed that cancer-associated peptides, as a cancer/testis antigen, can stimulate CTL. Thanks to the integration of biology and computer sciences, there are many available software and webservers that enable us to design and validate biological processes in a dry lab as in silico. Hence, we designed a candidate vaccine as a fusion protein, containing PLAC1, which is expressed highly in several types of tumor tissues, but not in normal ones, except placenta and testis ${ }^{[32]}$. PLAC1 is a suitable candidate for cancer immunotherapy, since it is overexpressed in more than $80 \%$ of breast cancers samples, while overexpression of Her2/neu, the target of Herceptin monoclonal antibody, is utmost $25 \%{ }^{[8,33]}$. Second part of this fusion protein vaccine is fliC from Salmonella enterica, which as an adjuvant molecule might be able to stimulate both humoral and cellular immune responses ${ }^{[34]}$. Third part of this construct is a flexible linker, GSGGSGGSGGSG, which resides between the mentioned parts and contains repeated small or polar amino acids (glycine and serine). Such linkers provide favorable solubility and flexibility and join two domains of fusion protein without changing their structure and function ${ }^{[35,36]}$.

The construct structure was analyzed and validated using diff erent bioinformatics tools and servers. Based on the results of different physicochemical parameters and structural analysis, PLAC1-fliC is a stable hydrophilic protein with a net negative charge. Its favorite degree of stability enables it to circulate easily and to be processed by antigen-presenting cells because proteins with a very loose conformation are susceptible to tertiary conformational epitope changes and might degrade easily. On the other hand, rigid structures might resist against antigen processing and epitope presentation ${ }^{[37]}$.

Secondary structures of polypeptides play an important role in their ultimate structure and function. Forecasting secondary structure from their amino acids

Table 2. Comparison of residues residence in Ramachandran plot before and after model refinement

\begin{tabular}{lcc}
\hline Database & Before refinement & After refinement \\
\hline RAMPAGE & & \\
Number of residues in & $603(86.9)$ & $640(92.2)$ \\
Favored region (\%) & $54(7.8)$ & $33(4.8)$ \\
Allowed region (\%) & $37(5.3)$ & $21(3.0)$ \\
Outlier region (\%) & & \\
& & \\
PROCHECK & $500(81.2)$ & $528(85.7)$ \\
Residues in & $81(13.1)$ & $63(10.2)$ \\
Most favored regions (\%) & $23(3.7)$ & $14(2.3)$ \\
Additional allowed regions (\%) & $12(1.9)$ & $11(1.8)$ \\
Generously allowed regions (\%) & & \\
Disallowed regions (\%) & & \\
\hline
\end{tabular}


Table 3. Antigenicity predictions of PLAC1, fliC, linker, and PLAC1-fliC.

\begin{tabular}{lcc}
\hline Protein & ANTIGENpro & VaxiJen $^{*}$ \\
\hline PLAC1 & 0.947422 & 0.6587 \\
fliC & 0.923670 & 0.8246 \\
linker & 0.453669 & 1.8025 \\
PLAC1-fliC & 0.955086 & 0.7320 \\
\hline
\end{tabular}

$*$ Threshold $=0.5$

sequences is the prerequisite for proteins 3D structure prediction, as well as for prediction of protein stability. GOR V server was used to predict the secondary structure of PLAC1-fliC. It works according to Garnier-Osguthorpe-Robson method that uses combined mathematical tools, probability methods, and data have been extracted from empirical methods like NMR and crystallography ${ }^{[38]}$. Table 1 reveals that the percentage of PLAC1-fliC random coil and alpha helix reduced and ioncreased, respectively. Therefore, its structure got more regular in comparison with PLAC1. Indeed, alpha helices are more stable than beta strands and random coils and more firm to conformational changes ${ }^{[39]}$. PLAC1-fliC modeling, refinement and validation were accomplished via different servers. Based on RAMPAGE results, most residues were located in allowed and favorite regions, and even the scores got better after refinement. These results were validated by PROCHECK, as well (Table 2). The predicted model of PLAC1-fliC, when compared with the experimental structures, indicated that this model was valid and matched with experimentally determined structures. Appropriate ProSA Z-scores confirms the accuracy of this model (Fig. 4) ${ }^{[20]}$.

Since the presence of flagella protein domain, as an adjuvant in the construct, might be allergenic for human; it is necessary to evaluate its potential allergenicity. It is important that vaccines could not be an allergen for humans. Allergens provoke hosts immune system and might lead to undesirable allergenic reactions ${ }^{[40]}$. The allergenicity prediction result showed that PLAC1-fliC was not allergen for humans; therefore, it can be administrated safely.

For stimulating immune responses against cancer cells and eventually eradicating them, their specific antigen epitopes have to be presented to immune cells, including $\mathrm{B}$ and $\mathrm{T}$ lymphocytes. Intrinsic antigens like cancer epitopes are presented to CTL by MHC-I, and extrinsic antigen such as adjuvants are presented to $\mathrm{T}$ helper cells by MHC-II molecules. Commonly, MHC-I presents 8-11 amino acids of peptides, while MHC-II presents peptides with 12-25 amino acids in length; they interact with T-cell receptor and eventually lead to cellular immune response ${ }^{[41]}$. The MHC genes are one of the most polymorphic human genes, and their frequency varies among populations, races, and ethnic groups. To predict the presenting potency and binding affinity of PLAC1-fliC in antigen-presenting cells, we selected the MHC-I and MHC-II alleles based on their global and regional relative haplotypes frequency according to scientific reports by investigating their frequency in allele frequency database ${ }^{[42-46]}$. As Table 4 and 5 show, immunodominant 9-mer peptide of PLAC1-fliC strongly binds to MHC-I haplotype HLAA0201, and 15-mer epitope of PLAC1-fliC binds to DRB1_ 0301 and DRB1_0401 MHC-II haplotypes. These results exhibit that PLAC1-fliC could be presented efficiently to immune cells and could evoke immunity against cancer cells. In addition, circulating antigens like protein-based vaccines could act as B-cell epitope and result in B-cell activation, antibody production, and humoral immunity stimulation. B-cell epitope is an antigenic determinant, recognized by antibodies or B-cell receptors. It can be a peptide, called the linear or continuous epitope, or it can have a 3D structure which is considered as a conformational or discontinuous epitope. B-cell epitopes of an antigen are antibody production stimulators ${ }^{[26]}$. Based on Tables 6-8, PLAC1-fliC has both linear and conformational epitopes with strong affinity to $\mathrm{B}$ and $\mathrm{T}$ cells; therefore, it could potentially evoke humoral and cellular immunity.

In conclusion, our investigation regarding probable vaccine for breast cancer reveals that PLAC1-fliC contains suitable structure and stability that could effectively stimulate both cellular and humoral immunity and might be safe to administer. Since our study is a bioinformatics analysis, experimental studies are warranted for validity of our results.

Table 4. HLA1-binding peptides based on NetMHC 4.0

\begin{tabular}{lccccc}
\hline Peptide sequence & Position & HLA & Affinity (nM) & Rank (\%) & Binding level \\
\hline FMLNNDVCV & 19 & HLA-A0201 & 7.53 & 0.07 & SB \\
FMVTVHPFM & 12 & HLA-A0201 & 10.81 & 0.12 & SB \\
SIDWFMVTV & 8 & HLA-A0201 & 26.82 & 0.40 & SB \\
VLCSIDWFM & 5 & HLA-A0201 & 35.00 & 0.50 & SB \\
\hline
\end{tabular}

$\mathrm{SB}$, strong binder 
Table 5. HLA2-binding peptides based on NetMHCII 2.3

\begin{tabular}{lccccc}
\hline Peptide sequence & \multirow{2}{*}{ Position } & HLA & $\begin{array}{c}\text { Affinity } \\
(\mathbf{n M})\end{array}$ & $\begin{array}{c}\text { Rank } \\
(\boldsymbol{\%})\end{array}$ & $\begin{array}{c}\text { Binding } \\
\text { level }\end{array}$ \\
\hline DTTIALDNSTFKASA & 1 & DRB1_0301 & 15.5 & 0.50 & SB* \\
ADTTIALDNSTFKAS & 2 & DRB1_0301 & 17.0 & 0.50 & SB \\
TTIALDNSTFKASAT & 3 & DRB1_0301 & 17.3 & 0.60 & SB \\
YADTTIALDNSTFKA & 4 & DRB1_0301 & 22.0 & 0.80 & SB \\
HPFMLNNDVCVHFHE & 5 & DRB1_0301 & 22.7 & 0.80 & SB \\
PFMLNNDVCVHFHEL & 6 & DRB1_0301 & 24.3 & 0.90 & SB \\
VHPFMLNNDVCVHFH & 7 & DRB1_0301 & 27.2 & 1.10 & SB \\
TIALDNSTFKASATG & 8 & DRB1_0301 & 33.6 & 1.40 & SB \\
FMLNNDVCVHFHELH & 9 & DRB1_0301 & 40.2 & 1.70 & SB \\
GYADTTIALDNSTFK & 10 & DRB1_0301 & 42.3 & 1.80 & SB \\
QNRFNSAITNLGNTV & 1 & DRB1_0401 & 6.3 & 0.01 & SB \\
VQNRFNSAITNLGNT & 2 & DRB1_0401 & 6.6 & 0.01 & SB \\
NRFNSAITNLGNTVN & 3 & DRB1_0401 & 7.3 & 0.03 & SB \\
AVQNRFNSAITNLGN & 4 & DRB1_0401 & 8.1 & 0.04 & SB \\
RFNSAITNLGNTVNN & 5 & DRB1_0401 & 11.7 & 0.12 & SB \\
GAVQNRFNSAITNLG & 6 & DRB1_0401 & 16.1 & 0.30 & SB \\
DSDYATEVSNMSRAQ & 7 & DRB1_0401 & 17.7 & 0.40 & SB \\
SDYATEVSNMSRAQI & 8 & DRB1_0401 & 19.0 & 0.40 & SB \\
EDSDYATEVSNMSRA & 9 & DRB1_0401 & 19.7 & 0.50 & SB \\
IEDSDYATEVSNMSR & 10 & DRB1_0401 & 27.9 & 0.90 & SB \\
DTTIALDNSTFKASA & 11 & DRB1_0401 & 29.5 & 1.00 & SB \\
DYATEVSNMSRAQIL & 12 & DRB1_0401 & 30.2 & 1.00 & SB \\
TTIALDNSTFKASAT & 13 & DRB1_0401 & 33.7 & 1.20 & SB \\
ADTTIALDNSTFKAS & 14 & DRB1_0401 & 33.9 & 1.20 & SB \\
VHPFMLNNDVCVHFH & 15 & DRB1_0401 & 42.5 & 1.70 & SB \\
GHNFKAQPDLAEAAT & 16 & DRB1_0401 & 44.9 & 1.80 & SB \\
TVHPFMLNNDVCVHF & 17 & DRB1_0401 & 45.4 & 1.90 & SB \\
RAQILQQAGTSVLAQ & 1 & DRB1_0101 & 8.3 & 1.90 & SB \\
\hline
\end{tabular}

*SB strong binder

Table 6. Predicted B-cell linear epitopes of PLAC1-fliC

\begin{tabular}{llllcc}
\hline No. & Start & End & Peptide & $\begin{array}{c}\text { Number of } \\
\text { residues }\end{array}$ & Score \\
\hline & & & QQKYKVSDTAATVTGYADTTIALDNSTFKASATGLGG & & \\
1 & 378 & 487 & $\begin{array}{l}\text { TDQKIDGDLKFDDTTGKYYAKVTVTGGTGKDGYY } \\
\text { EVSVDKTNGEVTLAGGATSPLTGGLPATATEDVKNVQVA }\end{array}$ & 110 & 0.815 \\
2 & 76 & 90 & IHYSSKGTPSKFVIP & 15 & 0.787 \\
3 & 14 & 51 & MVTVHPFMLNNDVCVHFHELHLGLGCPPNHVQPHAYQF & 38 & 0.778 \\
4 & 1 & 6 & QSPMTV & 6 & 0.74 \\
5 & 237 & 261 & GLRINSAKDDAAGQAIANRFTANIK & 25 & 0.726 \\
6 & 173 & 194 & LDISEDWSLHTDDMIGSMGSGG & 22 & 0.708 \\
7 & 679 & 696 & SVLAQANQVPQNVLSLLR & 18 & 0.69 \\
8 & 151 & 168 & HTQVPCHQAGAQEAQPLQ & 18 & 0.662 \\
9 & 513 & 523 & SYTDNNGKTID & 11 & 0.654 \\
10 & 107 & 123 & SMRVASKSRATAQKDEK & 17 & 0.649 \\
11 & 298 & 310 & AVQSANSTNSQSD & 13 & 0.637 \\
12 & 196 & 222 & GGSGGSGMAQVINTNSLSLLTQNNLNK & 27 & 0.61 \\
13 & 138 & 145 & NCDCPPCV & 8 & 0.593 \\
14 & 603 & 615 & ATTTENPLQKIDA & 13 & 0.588 \\
15 & 647 & 658 & NLTSARSRIEDS & 12 & 0.585 \\
16 & 347 & 359 & TIQVGANDGETID & 13 & 0.581 \\
\hline
\end{tabular}


Table 7. Predicted B-cell discontinuous epitopes of PLAC1-fliC

\begin{tabular}{|c|c|c|c|}
\hline No. & Residues & $\begin{array}{c}\text { Number of } \\
\text { residues }\end{array}$ & Score \\
\hline 1 & 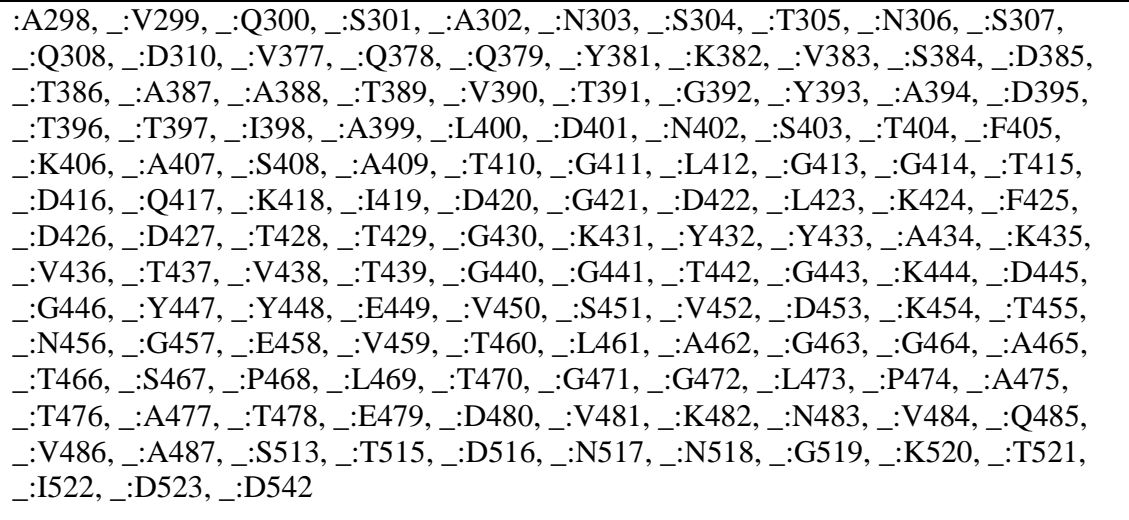 & 133 & 0.787 \\
\hline 2 & 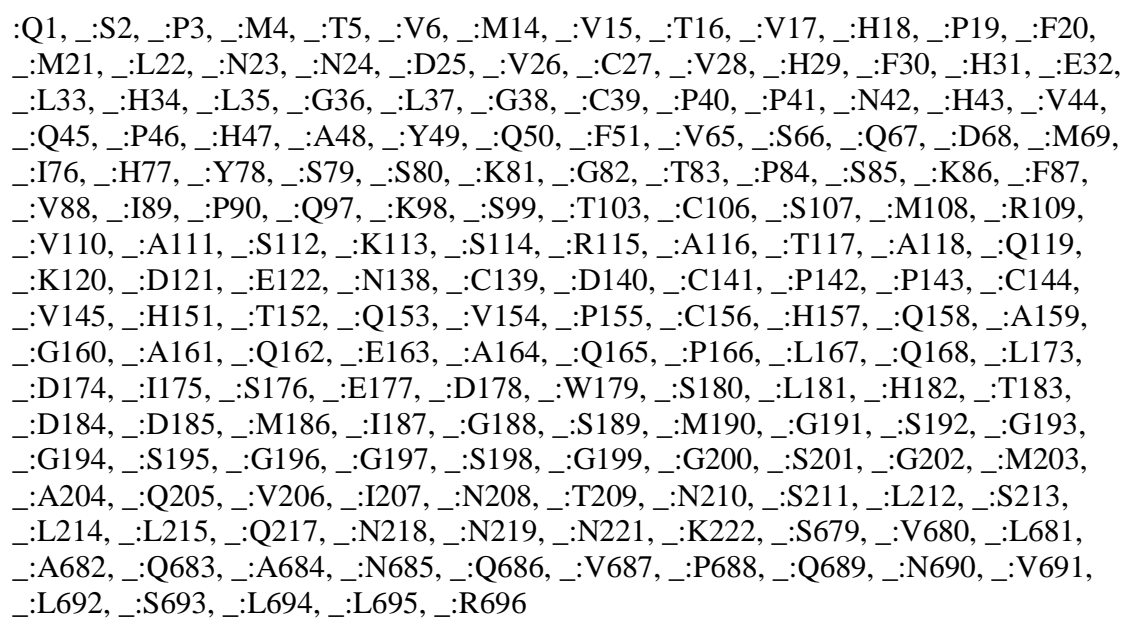 & 177 & 0.687 \\
\hline 3 & 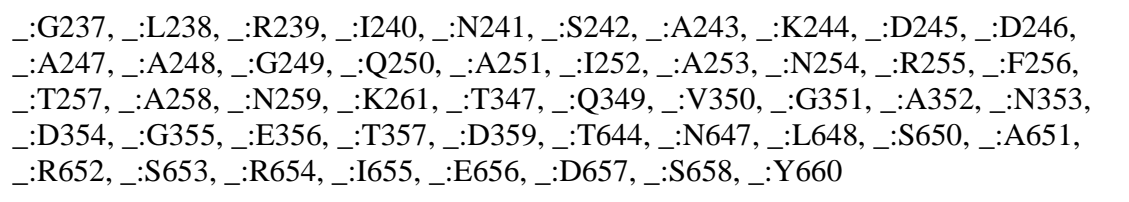 & 48 & 0.667 \\
\hline 4 & $\begin{array}{l}\text { _:G371, _:L372, _:L375,_:A569,_:A602, _:A603,_:T604, _:T605,_:T606,_:E607, } \\
\text { _:N608,_:P609,_:L610,_:Q611,_:K612 }\end{array}$ & 15 & 0.500 \\
\hline
\end{tabular}

Table 8. Predicted CTL epitopes of PLAC1-fliC

\begin{tabular}{cccc}
\hline $\begin{array}{c}\text { Peptide } \\
\text { rank }\end{array}$ & $\begin{array}{c}\text { Start } \\
\text { position }\end{array}$ & Sequence & Score (ANN/SVM) $^{*}$ \\
\hline 1 & 9 & SIDWFMVTV & $0.73 / 1.2411843$ \\
2 & 36 & GLGCPPNHV & $0.96 / 0.71105708$ \\
3 & 61 & RAKAVSQDM & $0.97 / 0.5147798$ \\
4 & 83 & TPSKFVIPV & $0.88 / 0.5965804$ \\
5 & 56 & TECGIRAKA & $0.85 / 0.5788711$ \\
6 & 87 & FVIPVSCAA & $0.94 / 0.37276101$ \\
7 & 70 & VIYSTEIHY & $0.82 / 0.4671223$ \\
8 & 22 & LNNDVCVHF & $0.57 / 0.59104808$ \\
9 & 119 & QKDEKCYEV & $0.95 / 0.14603929$ \\
10 & 6 & VLCSIDWFM & $0.46 / 0.53298459$ \\
\hline
\end{tabular}

${ }^{*}$ Cut-off score: $0.51(\mathrm{ANN})$ and 0.36 (SVM); ANN, artificial neural network; SVM, support vector machine 


\section{ACKNOWLEDGEMENTS}

This study was financially supported by the Office of Vice-Chancellor for Research of Shiraz University of Medical Sciences, Shiraz, Iran (Grant No. 98-01-3619977). The authors wish to thank Mr. H. Argasi at the Research Consultation Center (RCC) of Shiraz University of Medical Sciences for his invaluable assistance in editing this manuscript.

CONFLICT OF INTEREST. None declared.

\section{REFERENCES}

1. Bray F, Ferlay J, Soerjomataram I, Siegel RL, Torre LA, Jemal A. Global cancer statistics 2018: GLOBOCAN estimates of incidence and mortality worldwide for 36 cancers in 185 countries. CA: a cancer journal for clinicians 2018; 68(6): 394-424.

2. Zhou J, Zhong Y. Breast cancer immunotherapy. Cellular and molecular immunology 2004; 1(4): 247255.

3. Song $\mathrm{Q}$, Zhang $\mathrm{CD}, \mathrm{Wu} \mathrm{XH}$. Therapeutic cancer vaccines: From initial findings to prospects. Immunology letters 2018; 196: 11-21.

4. Chang WL, Yang Q, Zhang H, Lin HY, Zhou Z, Lu X, Zhu C, Xue LQ, Wang H. Role of placenta-specific protein 1 in trophoblast invasion and migration. Reproduction 2014; 148(4): 343-352.

5. Kulkarni P, Shiraishi T, Rajagopalan K, Kim R, Mooney SM, Getzenberg RH. Cancer/testis antigens and urological malignancies. Nature reviews urology 2012; 9(7): 386-396.

6. Afsharpad M, Nowroozi MR, Ayati M, Saffari M, Nemati S, Mohebbi E, Nekoohesh L, Zendehdel K, Modarressi MH. ODF4, MAGEA3, and MAGEB4: Potential Biomarkers in Patients with Transitional Cell Carcinoma. Iranian biomedical journal 2018; 22(3): 160-170.

7. Li Y, Chu J, Li J, Feng W, Yang F, Wang Y, Zhang Y, Sun C, Yang M, Vasilatos SN, Huang Y, Fu Z, Yin Y. Cancer/testis antigen- Plac1 promotes invasion and metastasis of breast cancer through Furin/NICD/PTEN signaling pathway. Molecular oncology 2018; 12(8): 1233-1248.

8. Koslowski M, Sahin U, Mitnacht-Kraus R, Seitz G, Huber C, Türeci Ö. A placenta-specific gene ectopically activated in many human cancers is essentially involved in malignant cell processes. Cancer research 2007; 67(19): 9528-9534.

9. Yuan H, Chen V, Boisvert M, Isaacs C, Glazer RI. PLAC1 as a serum biomarker for breast cancer. PLoS one 2018; 13(2): e0192106

10. Hekmat S, Sadat SM, Aslani MM, Mahdavi M, Bolhassani A, Asgar Halvaee F, Ghahari SMM, Aghasadeghi MR, Siadat SD. Truncated core/NS3 fusion protein of $\mathrm{HCV}$ adjuvanted with outer membrane vesicles of Neisseria meningitidis serogroup B: Potent inducer of the murine immune system. Iranian biomedical journal 2019; 23(4): 235-245.

11. Cui B, Liu X, Fang Y, Zhou P, Zhang Y, Wang Y. Flagellin as a vaccine adjuvant. Expert review of vaccines 2018; 17(4): 335-349.

12. Atapour A, Mostafavi-Pour Z, Mokarram P, Mohammadi S. Cloning, expression and purification of a novel fusion protein composed of flagellin and NS5B of hepatitis C virus in Escherichia coli host. Shiraz, Emedical journal 2018; 20(1): e79898.

13. Khani MH, Bagheri M, Zahmatkesh A, Bidhendi SM. Immunostimulatory effects of truncated and full-length flagellin recombinant proteins. Microbial pathogenesis 2019; 127: 190-197.

14. Yoon SI, Kurnasov O, Natarajan V, Hong M, Gudkov AV, Osterman AL, Wilson IA. Structural basis of TLR5-flagellin recognition and signaling. Science 2012; 335(6070): 859-864.

15. Song L, Xiong D, Kang X, Yang Y, Wang J, Guo Y, Xu $H$, Chen S, Peng D, Pan Z, Jiao X. An avian influenza A (H7N9) virus vaccine candidate based on the fusion protein of hemagglutinin globular head and Salmonella typhimurium flagellin. BMC biotechnology 2015; 15(1): 79.

16. Gasteiger E, Hoogland C, Gattiker A, Duvaud S, Wilkins MR, Appel RD, Bairoch A. Protein Identification and Analysis Tools on the ExPASy Server. The proteomics protocols handbook. Germany: Springer; 2005.

17. Sen TZ, Jernigan RL, Garnier J, Kloczkowski A. GOR $\mathrm{V}$ server for protein secondary structure prediction. Bioinformatics 2005; 21(11): 2787-2788.

18. Kelley LA, Mezulis S, Yates CM, Wass MN, Sternberg MJ. The Phyre2 web portal for protein modeling, prediction and analysis. Nature protocols 2015; 10(6): 845-858.

19. Lee GR, Heo L, Seok C. Effective protein model structure refinement by loop modeling and overall relaxation. Proteins 2016; 84(Suppl 1): 293-301.

20. Wiederstein M, Sippl MJ. ProSA-web: interactive web service for the recognition of errors in three-dimensional structures of proteins. Nucleic acids research 2007; 35(Web Server issue): W407-W410.

21. Laskowski RA, MacArthur MW, Moss DS, Thornton JM. PROCHECK: a program to check the stereochemical quality of protein structures. Journal of applied crystallography 1993; 26(2): 283-291.

22. Doytchinova IA, Flower DR. VaxiJen: a server for prediction of protective antigens, tumour antigens and subunit vaccines. BMC bioinformatics 2007; 8: 4.

23. Dimitrov I, Naneva L, Doytchinova I, Bangov I. AllergenFP: allergenicity prediction by descriptor fingerprints. Bioinformatics 2014; 30(6): 846-851.

24. Andreatta M, Nielsen M. Gapped sequence alignment using artificial neural networks: application to the MHC class I system. Bioinformatics 2015; 32(4): 511-517.

25. Jensen KK, Andreatta M, Marcatili P, Buus S, Greenbaum JA, Yan Z, Sette A, Peters B, Nielsen M. Improved methods for predicting peptide binding affinity to MHC class II molecules. Immunology 2018; 
154(3): 394-406.

26. Ponomarenko J, Bui HH, Li W, Fusseder N, Bourne PE, Sette A, Peters B. ElliPro: a new structure-based tool for the prediction of antibody epitopes. BMC bioinformatics 2008; 9(1): 514.

27. Bhasin M, Raghava G. Prediction of CTL epitopes using QM, SVM and ANN techniques. Vaccine 2004; 22(2324): 3195-3204.

28. Hutchinson L. Breast cancer: challenges, controversies, breakthroughs. Nature reviews clinical oncology 7(2): 669-670.

29. Vergati M, Intrivici C, Huen NY, Schlom J, Tsang KY. Strategies for cancer vaccine development. BioMed research international 2010; 2010" Article ID 596432.

30. Kawada J, Wada $H$, Isobe $M$, Gnjatic S, Nishikawa $H$, Jungbluth AA, Okazaki N, Uenaka A, Nakamura Y, Fujiwara S, Mizuno N, Saika T, Ritter E, Yamasaki M, Miyata H, Ritter G, Murphy R, Venhaus R, Pan L, Old LJ, Doki Y, Nakayama E. Heteroclitic serological response in esophageal and prostate cancer patients after NY-ESO-1 protein vaccination. International journal of cancer 2012; 130(3): 584-592.

31. Shim E, Shim H, Bae J, Lee H, Jeoung D. CAGE displays oncogenic potential and induces cytolytic $\mathrm{T}$ lymphocyte activity. Biotechnology letters 2006; 28(7): 515-522.

32. Silva WA Jr., Gnjatic S, Ritter E, Chua R, Cohen T, Hsu M, Jungbluth AA, Altorki NK, Chen YT, Old LJ, Simpson AJG, Caballero OL. PLAC1, a trophoblastspecific cell surface protein, is expressed in a range of human tumors and elicits spontaneous antibody responses. Cancer immunity 2007; 7: 18.

33. Slamon DJ, Godolphin W, Jones LA, Holt JA, Wong SG, Keith DE, Levin WJ, Stuart SG, Udove J, Ullrich A. Studies of the HER-2/neu proto-oncogene in human breast and ovarian cancer. Science 1989; 244(4905): 707-712.

34. Delavari S, Sohrabi M, Ardestani MS, Faezi S, Tebianian M, Taghizadeh M, Shajiei A, Hosseini SY, Moghaddampour M, Mahdavi M. Pseudomonas aeruginosa flagellin as an adjuvant: superiority of a conjugated form of flagellin versus a mixture with a human immunodeficiency virus type 1 vaccine candidate in the induction of immune responses. Journal of medical microbiology 2015; 64(11): 1361-1368.

35. Chen X, Zaro JL, Shen WC. Fusion protein linkers: property, design and functionality. Advanced drug delivery reviews 2013; 65(10): 1357-1369.

36. Hachikubo $\mathrm{Y}$, Ito $\mathrm{K}$, Schiefelbein J, Manstein DJ,
Yamamoto K. Enzymatic activity and motility of recombinant Arabidopsis myosin XI, MYA1. Plant and cell physiology 2007; 48(6): 886-891.

37. Scheiblhofer S, Laimer J, Machado Y, Weiss R, Thalhamer J. Influence of protein fold stability on immunogenicity and its implications for vaccine design. Expert review of vaccines 2017; 16(5): 479-489.

38. Kouza M, Faraggi E, Kolinski A, Kloczkowski A. The GOR method of protein secondary structure prediction and its application as a protein aggregation prediction tool. Methods in molecular biology 2017; 1484: 7-24.

39. Abrusán G, Marsh JA. Alpha helices are more robust to mutations than beta strands. PLoS computational biology 2016; 12(12): e1005242.

40. Kelso JM, Greenhawt MJ, Li JT, Nicklas RA, Bernstein DI, Blessing-Moore J, Cox L, Khan D, Lang DM, Oppenheimer J, Portnoy JM, Randolph C, Schuller DE, Spector SL, Tilles SA, Wallace D. Adverse reactions to vaccines. Annals of allergy, asthma and immunology 2009; 103(1): 25-43.

41. Neefjes J, Jongsma ML, Paul P, Bakke O. Towards a systems understanding of MHC class I and MHC class II antigen presentation. Nature reviews immunology 2011; 11(12): 823-836.

42. Amirzargar A, Mytilineos J, Farjadian S, Doroudchi M, Scherer S, Opelz G, Ghaderi A. Human leukocyte antigen class II allele frequencies and haplotype association in Iranian normal population. Human immunology 2001; 62(11): 1234-1238.

43. González-Galarza FF, Takeshita LY, Santos EJ, Kempson F, Maia MHT, da Silva AL, Teles e Silva AL, Ghattaoraya GS, Alfirevic A, Jones AR, Middleton D. Allele frequency net 2015 update: new features for HLA epitopes, KIR and disease and HLA adverse drug reaction associations. Nucleic acids research 2014; 43(Database issue): D784-D788.

44. Esmaeili A, Rabe SZT, Mahmoudi M, Rastin M. Frequencies of HLA-A, B and DRB1 alleles in a large normal population living in the city of Mashhad, Northeastern Iran. Iranian journal of basic medical sciences 2017; 20(8): 940-943.

45. Varzi AM, Shahsavar F, Tarrahi MJ. Distribution of HLA-DRB1 and HLA-DQB1 alleles in Lak population of Iran. Human immunology 2016; 77(7): 580-583.

46. Moatter T, Aban M, Tabassum S, Shaikh U, Pervez S. Molecular analysis of human leukocyte antigen class I and class II allele frequencies and haplotype distribution in Pakistani population. Indian journal of human genetics 2010; 16(3): 149-153. 